\title{
Mean-field anticipated BSDEs driven by time-changed Lévy noises
}

Youxin Liu' ${ }^{1,2^{*}}$ (D) and Yang Dai ${ }^{3}$

\section{"Correspondence:}

youxinliu@126.com

yxliu@whit.edu.cn

'Department of Elementary

Teaching, Wuhu Institute of

Technology, Wuhu, China

${ }^{2}$ School of Science, Nanjing University of Science and

Technology, Nanjing, China Full list of author information is available at the end of the article

\begin{abstract}
The objective of this work is to show a new kind of mean-field anticipated backward stochastic differential equation (in short MF-ABSDE) driven by time-changed Lévy noises. We give two methods to prove the existence and uniqueness of the solution of those equations by the fixed point theorem and the Picard iterative sequence. Finally, we obtain a comparison theorem for the solutions.
\end{abstract}

Keywords: Mean-field limits; Anticipated BSDE; Time-changed Lévy process; Comparison theorem

\section{Introduction}

With the pioneering work of Pardoux and Peng [13], different properties of backward stochastic differential equations (in short BSDEs) in wider areas have attracted many researchers' great interests. Applying these results to finance, biology and physics, such processes appear in many different applications. Many achievements have been made in the research of BSDEs in a more general framework. We note there are several constructions of BSDEs in the literature. See e.g. El Karou et al. [5], Peng [14], Buckdahn et al. [2] and Douissi et al. [4]. In mathematical terms, following for instance Peng and Yang [15], this amounts to solving a fundamental class of BSDE, called anticipated BSDE; that is,

$$
\left\{\begin{array}{l}
Y_{t}=\xi_{T}+\int_{t}^{T} f\left(s, Y_{s}, Z_{s}, Y_{s+u(s)}, Z_{s+v(s)}\right) d s-\int_{t}^{T} Z_{s} d W_{s} \\
Y_{t}=\xi_{t}, \quad t \in[T, T+K] \\
Z_{t}=\eta_{t}, \quad t \in[T, T+K]
\end{array}\right.
$$

where $u(t), v(t) \in C[0, T]$ and they satisfy

(A1) $\exists K>0, \forall t \in[0, T]$ such that

$$
t+u(t) \leq T+K, \quad t+v(t) \leq T+K
$$

(A2) $\exists L>0, \forall t \in[0, T], g(t) \geq 0$ and $g(t) \in L[0, T+K]$ such that

$$
\int_{t}^{T} g(s+u(s)) d s \leq L \int_{t}^{T+K} g(s) d s, \quad \int_{t}^{T} g(s+v(s)) d s \leq L \int_{t}^{T+K} g(s) d s .
$$

(c) The Author(s) 2020. This article is licensed under a Creative Commons Attribution 4.0 International License, which permits use, sharing, adaptation, distribution and reproduction in any medium or format, as long as you give appropriate credit to the original author(s) and the source, provide a link to the Creative Commons licence, and indicate if changes were made. The images or other third party material in this article are included in the article's Creative Commons licence, unless indicated otherwise in a credit line to the material. If material is not included in the article's Creative Commons licence and your intended use is not permitted by statutory regulation or exceeds the permitted use, you will need to obtain permission directly from the copyright holder. To view a copy of this licence, visit http://creativecommons.org/licenses/by/4.0/ 
Clearly we note that the generator $f(\cdot)$ contains many values $\left(Y_{t}, Z_{t}\right)$ of current time and future time in Eq. (1). And the authors dealt with the fact that the anticipated BSDE has a unique result and established a comparison theorem for the solution. Also, they verified by a duality relationship between anticipated BSDEs and stochastic differential delay equations. Using the duality, they solved some stochastic optimal control problems (see e.g. Xu [20], Yu [21], Giulia and Steffen [7], Klimsiak [9], Wang [19], Wang, Shi and Meng [18], Zhang and Yan [22], among others).

Furthermore, Lu and Ren [12] studied anticipated backward stochastic differential equations on Markov chains. Richter [17] studied a rich and flexible kind of quadratic BSDEs involving affine processes and explicated up to the solution of an ODE in detail. Frei [6] established a new notion of local solution ofq BSDEs and quoted the implications in a financial context. Based on the work of Ren and Mohamed [16], Kallsen and Muhle-Karbe [8], Di Nunno and Sjursen (2014) [3] studied a kind of BSDEs when the noises are timechanging Lévy noises and the generator $f(\cdot)$ is linear. In the article they also gave the meanvariance portfolio selection.

Very recently, Liu and Ren [11] considered anticipated BSDE for time-changed Lévy noises of the type

$$
\left\{\begin{array}{l}
Y_{t}=\xi_{T}+\int_{t}^{T} f\left(s, \lambda_{s}, Y_{s}, Z_{s}, Y_{s+u(s)}, Z_{s+\nu(s)}\right) d s-\int_{t}^{T} Z_{s}(x) \mu(d s, d x) \\
Y_{t}=\xi_{t}, \quad t \in[T, T+K] \\
Z_{t}=\eta_{t}, \quad t \in[T, T+K]
\end{array}\right.
$$

where $\mu$ is the structure of the mixture of a conditional Brownian measure and a doubly stochastic Poisson measure as follows:

$$
\mu(\alpha):=B(\alpha \cap[0, T] \times\{0\})+\tilde{H}(\alpha \cap[0, T] \times\{\mathbb{R} \backslash\{0\}\}) .
$$

They talked about the classical problem of the solutions in depth and showed the connection between the two kinds of equations. Liu [10] continued the study of these equations. He gave a direct proof using useful a priori estimates of the solution and included some applications of the classical Feynman-Kac formula.

On the other hand, mean-field limits have played a very important role in different fields of physics and chemistry, but have found in recent work also application in economics and game theory (Buckdahn et al. [2]). Specifically, as far as we are aware of, mean-field limits also have been researched in the context of their applications, such as the optimal control problem, the McKean-Vlasov equation and stochastic games. More and more scholars begin to study stochastic systems with mean-field interaction. Buckdahn et al. [2] investigated mean-field SDEs associated McKean-Vlasov forward SPEs and PDEs. Buckdahn et al. [1] studied the optimal control problem for a kind of general mean-field SDEs. Douissi et al. [4] researched a related stochastic optimal control problem of MF-BSDEs when the noises are fractional Brownian. 
In our approach here we are concerned with a kind of MF-ABSDE driven by timechanging Lévy noises, it follows that

$$
\left\{\begin{aligned}
Y_{t}= & \xi_{T}+\int_{t}^{T} E^{\prime}\left[f\left(s, \lambda_{s}, Y_{s}, Z_{s}, Y_{s}^{\prime}, Z_{s}^{\prime}, Y_{s+u(s)}, Z_{s+v(s)}, Y_{s+u(s)}^{\prime}, Z_{s+v(s)}^{\prime}\right)\right] d s \\
& -\int_{t}^{T} Z_{s}(x) \mu(d s, d x) \\
Y_{t}= & \xi_{t}, \quad t \in[T, T+K] \\
Z_{t}= & \eta_{t}, \quad t \in[T, T+K] .
\end{aligned}\right.
$$

The main objective of this manuscript is the profound study of the anticipated BSDEs (e.g. Liu (2016), [10]). We study the equation in the sense of mean-field limits. In other words, the aim of this manuscript is to discuss some applications of the mean-field anticipated backward stochastic differential equation driven by time-changing Lévy noises. One of the motivations is to study the solution of MF-ABSDE (3). And we give two methods to prove the existence and uniqueness of this solution. Finally, we explore the comparison theorem.

An outline of the paper is as follows: Sect. 2 is devoted to recalling of concepts and auxiliary results. In Sect. 3, we present the existence and uniqueness of the solution for the MF-ABSDE with two methods. In Sect. 4, we obtain the comparison theorem of the solutions.

\section{The framework}

The aim of this section is to present some concepts and notation. For more details, one can see Buckdahn, Li and Peng [2], Di Nunno and Sjursen [3], Douissi, Wen and Shi [4] and the references therein.

Suppose $X:=[0, T] \times \mathbb{R}=([0, T] \cup\{0\}) \cup([0, T] \times\{\mathbb{R} \backslash\{0\}\})$. and let $(\bar{\Omega}, \overline{\mathcal{F}}, \bar{P}):=(\Omega \times$ $\Omega, \mathcal{F} \otimes \mathcal{F}, P \otimes P)$ be the product of $(\Omega, \mathcal{F}, P)$ with itself. Suppose $T>0$, let $\lambda_{t}:=\left(\lambda_{t}^{B}, \lambda_{t}^{H}\right) \geq 0$ be a two-dimensional stochastic process satisfying

(B1) $\forall \epsilon>0, \forall t \in[0, T], \lim _{\Delta t \rightarrow 0} P\left(\left|\lambda_{t+\Delta t}^{k}-\lambda_{t}^{k}\right| \geq \epsilon\right)=0, k=B, H$;

(B2) $E\left[\int_{0}^{T} \lambda_{t}^{k} d t\right]<+\infty, k=B, H$.

Consider the following mixture of measures:

$$
\begin{aligned}
\Lambda(\alpha) & :=\int_{0}^{T} \mathbf{1}_{\{(s, 0) \in \alpha\}}(s) \lambda_{s}^{B} d s+\int_{0}^{T} \int_{\mathbb{R}_{0}} \mathbf{1}_{\alpha}(s, x) q(d x) \lambda_{s}^{H} d s \\
& =\Lambda^{B}(\alpha \cap[0, T] \times\{0\})+\Lambda^{H}(\alpha \cap[0, T] \times\{\mathbb{R} \backslash\{0\}\}),
\end{aligned}
$$

where $\alpha \subseteq X$, and $q$ is a deterministic, $\sigma$-finite measure on $\mathcal{B}\{\mathbb{R} \backslash\{0\}\}$ such that

$$
\int_{\mathbb{R}_{0}} z^{2} q(d x)<+\infty
$$

Let we now define the noises driving (3),

Definition $1 B$ is a random measure on $\mathcal{B}\{[0, T] \times\{0\}\} . H$ is a random measure on $\mathcal{B}\{[0, T] \times\{\mathbb{R} \backslash\{0\}\}$. They satisfy $(\mathrm{C} 1)-(\mathrm{C} 5)$.

(C1) $P\left(B(\alpha) \leq t \mid \mathcal{F}^{\Lambda}\right)=P\left(B(\alpha) \leq t \mid \Lambda^{B}(\alpha)\right)=\Phi\left(\frac{t}{\sqrt{\Lambda^{B}(\alpha)}}\right), t \in \mathbb{R}, \alpha \subseteq[0, T] \times\{0\}$, where $\Phi(\cdot)$ represents the density function of the standard normal distribution function. 
(C2) $P\left(B\left(\alpha_{1}\right) \cap B\left(\alpha_{2}\right) \mid \mathcal{F}^{\Lambda}\right)=P\left(B\left(\alpha_{1}\right) \mid \mathcal{F}^{\Lambda}\right) \cap P\left(B\left(\alpha_{2}\right) \mid \mathcal{F}^{\Lambda}\right)$ whenever $\alpha_{1} \cap \alpha_{2}=\phi$, where $\alpha_{1}, \alpha_{2} \subseteq[0, T] \times\{0\}$.

(C3) $P\left(H(\alpha)=k \mid \mathcal{F}^{\Lambda}\right)=P\left(H(\alpha)=k \mid \Lambda^{H}(\alpha)\right)=\frac{\Lambda^{H}(\alpha)^{k}}{k !} e^{-\Lambda^{H}(\alpha)}, k \in \mathbb{N}$, $\alpha \subseteq[0, T] \times\{\mathbb{R} \backslash\{0\}\}$.

(C4) $P\left(H\left(\alpha_{1}\right) \cap H\left(\alpha_{2}\right) \mid \mathcal{F}^{\Lambda}\right)=P\left(H\left(\alpha_{1}\right) \mid \mathcal{F}^{\Lambda}\right) \cap P\left(H\left(\alpha_{2}\right) \mid \mathcal{F}^{\Lambda}\right)$ whenever $\alpha_{1} \cap \alpha_{2}=\phi$, where $\alpha_{1}, \alpha_{2} \subseteq[0, T] \times\{\mathbb{R} \backslash\{0\}\}$.

(C5) $B$ and $H$ are conditionally independent given $\mathcal{F}^{\Lambda}$.

Suppose $\tilde{H}:=H-\Lambda^{H}$ is the signed random measure of the form

$$
\tilde{H}(\alpha)=H(\alpha)-\Lambda^{H}(\alpha), \quad \alpha \subset[0, T] \times\{\mathbb{R} \backslash\{0\}\} .
$$

Definition 2 We define the singed random measure $\mu$ on the Borel subsets of X by

$$
\mu(\alpha):=B(\alpha \cap[0, T] \times\{0\})+\tilde{H}(\alpha \cap[0, T] \times\{\mathbb{R} \backslash\{0\}\}), \quad \alpha \subseteq X
$$

Clearly, from (C1)-(C5), we get

$$
\begin{aligned}
& \mathbb{E}\left[B(\alpha) \mid \mathcal{F}^{\Lambda}\right]=0, \quad \mathbb{E}\left[\tilde{H}(\alpha) \mid \mathcal{F}^{\Lambda}\right]=0, \quad \mathbb{E}\left[\mu(\alpha) \mid \mathcal{F}^{\Lambda}\right]=0, \\
& \mathbb{E}\left[B(\alpha)^{2} \mid \mathcal{F}^{\Lambda}\right]=\Lambda^{B}(\alpha), \quad \mathbb{E}\left[\tilde{H}(\alpha)^{2} \mid \mathcal{F}^{\Lambda}\right]=\Lambda^{H}(\alpha), \quad \mathbb{E}\left[\mu(\alpha)^{2} \mid \mathcal{F}^{\Lambda}\right]=\Lambda(\alpha),
\end{aligned}
$$

and

$$
\mathbb{E}\left[\mu\left(\alpha_{1}\right) \mu\left(\alpha_{2}\right) \mid \mathcal{F}^{\Lambda}\right]=\mathbb{E}\left[\mu\left(\alpha_{1}\right) \mid \mathcal{F}^{\Lambda}\right] \mathbb{E}\left[\mu\left(\alpha_{2}\right) \mid \mathcal{F}^{\Lambda}\right]=0,
$$

where $\alpha_{1} \cap \alpha_{2}=\phi$. So $\alpha_{1}$ and $\alpha_{2}$ are orthogonal given $\mathcal{F}^{\Lambda}$. The random measure $B$ and $H$ are related to a specific form of time change for Brownian motion and a pure Lévy process. To be more specific, for convenience, we define $\Lambda_{t}^{B}:=\int_{0}^{t} \lambda_{s}^{B} d s$ and $\tilde{\lambda}_{t}^{H}:=\int_{0}^{t} \lambda_{s}^{H} d s$, $t \in[0, T]$.

Set $\mathbb{F}=\left\{\mathcal{F}_{t}, t \in[0, T]\right\}$, where $\mathcal{F}_{t}=\bigcap_{r>t} \mathcal{F}_{r}^{\mu}$ and $\mathcal{F}_{t}^{\mu}=\mathcal{F}_{t}^{B} \vee \mathcal{F}_{t}^{H} \vee \mathcal{F}_{t}^{\Lambda}$. Furthermore, we set $\mathbb{G}=\left\{\mathcal{G}_{t}, t \in[0, T]\right\}$ with $\mathcal{G}_{t}=\mathcal{F}_{t}^{\mu} \vee \mathcal{F}^{\Lambda}$. Indeed it is not a natural choice of filtration because it includes some anticipating information, the future values of $\Lambda^{B}$ and $\Lambda^{H}$.

We give this product space to the filtration $\overline{\mathbb{F}}=\left\{\overline{\mathcal{F}}_{t}=\mathcal{F} \otimes \mathcal{F}_{t}, t \in[0, T]\right\}$. A random variable $\xi \in L^{1}(\Omega, \mathcal{F}, P)$ originally defined on $\Omega$ is extended canonically to $\bar{\Omega}: \xi^{\prime}\left(\omega^{\prime}, \omega\right)=\xi\left(\omega^{\prime}\right)$, $\left(\omega^{\prime}, \omega\right) \in \bar{\Omega}=\Omega \times \Omega$. For $\forall \theta \in(\bar{\Omega}, \overline{\mathcal{F}}, \bar{P})$, the variable $\theta(\cdot, \omega): \Omega \rightarrow \mathbb{R}$ is in $L^{1}(\Omega, \mathcal{F}, P), \mathbb{P}$-a.s. and its expectation is denoted by

$$
E^{\prime}[\theta(\cdot, \omega)]=\int_{\Omega} \theta\left(\omega^{\prime}, \omega\right) P\left(d \omega^{\prime}\right) .
$$

Hence we have $E^{\prime}[\theta]=E^{\prime}[\theta(\cdot, \omega)] \in L^{1}(\Omega, \mathcal{F}, P)$ and

$$
\bar{E}[\theta(\cdot, \omega)]=\int_{\bar{\Omega}} \theta(\cdot, \omega) d \bar{P}=\int_{\Omega} E^{\prime}[\theta(\cdot, \omega)] P(d \omega)=E\left[E^{\prime}(\theta)\right] .
$$

Remark 3 For our notation, we consider the following derivation:

$$
\begin{aligned}
E^{\prime} & {\left[f\left(t, \lambda_{t}, Y_{t}, Z_{t}, Y_{t}^{\prime}, Z_{t}^{\prime}, Y_{t+u(t)}, Z_{t+v(t)}, Y_{t+u(t)}^{\prime}, Z_{t+v(t)}^{\prime}\right)\right](\omega) } \\
& =E^{\prime}\left[f\left(t, \lambda_{t}, Y_{t}(\omega), Z_{t}(\omega), Y_{t}^{\prime}, Z_{t}^{\prime}, Y_{t+u(t)}(\omega), Z_{t+v(t)}(\omega), Y_{t+u(t)}^{\prime}, Z_{t+v(t)}^{\prime}\right)\right]
\end{aligned}
$$




$$
\begin{aligned}
= & \int_{\Omega}\left[f \left(t, \lambda_{t}, Y_{t}(\omega), Z_{t}(\omega), Y_{t}^{\prime}\left(\omega^{\prime}\right), Z_{t}^{\prime}\left(\omega^{\prime}\right), Y_{t+u(t)}(\omega), Z_{t+v(t)}(\omega), Y_{t+u(t)}^{\prime}\left(\omega^{\prime}\right),\right.\right. \\
& \left.\left.Z_{t+v(t)}^{\prime}\left(\omega^{\prime}\right)\right)\right] P\left(d \omega^{\prime}\right) .
\end{aligned}
$$

In addition,

$$
\begin{aligned}
E\left\{E^{\prime}\left[f\left(t, \lambda_{t}, Y_{t}^{\prime}, Z_{t}^{\prime}, Y_{t+u(t)}^{\prime}, Z_{t+v(t)}^{\prime}\right)\right]\right\} & =E\left\{E\left[f\left(t, \lambda_{t}, Y_{t}, Z_{t}, Y_{t+u(t)}, Z_{t+v(t)}\right)\right]\right\} \\
& =E\left[f\left(t, \lambda^{t}, Y_{t}, Z_{t}, Y_{t+u(t)}, Z_{t+v(t)}\right)\right] ; \\
E\left\{E^{\prime}\left[f\left(t, \lambda_{t}, Y_{t}, Z_{t}, Y_{t+u(t)}, Z_{t+v(t)}\right)\right]\right\} & =E\left\{E\left[f\left(t, \lambda_{t}, Y_{t}, Z_{t}, Y_{t+u(t)}, Z_{t+v(t)}\right)\right]\right\} \\
& =E\left[f\left(t, \lambda_{t}, Y_{t}, Z_{t}, Y_{t+u(t)}, Z_{t+v(t)}\right)\right] .
\end{aligned}
$$

Here and in the sequel, we shall denote the following two appropriate spaces of processes which are used frequently:

- $S_{\mathbb{G}}^{2}(0, T+K ; \mathbb{R}):=\left\{\phi_{t}(\omega)_{(0 \leq t \leq T+K, \omega \in \Omega)}\right.$ real-valued $\mathbb{G}$-adapted stochastic process:

$$
\left.\left(\mathbb{E}\left[\sup _{0 \leq t \leq T+K}\left|\phi_{t}(\omega)\right|^{2}\right]\right)^{1 / 2}<+\infty\right\} .
$$

- $L_{\mathbb{G}}^{2}(0, T+K ; \mathbb{R}):=\left\{\varphi_{t}(\omega)_{(0 \leq t \leq T+K, \omega \in \Omega)}\right.$ real-valued $\mathbb{G}$-adapted stochastic process:

$$
\begin{aligned}
& \left(\mathbb{E}\left[\int_{0}^{T+K}\left|\varphi_{s}(0)\right|^{2} \lambda_{s}^{B} d s+\int_{0}^{T+K} \int_{\mathbb{R}_{0}}\left|\varphi_{s}(x)\right|^{2} q(d x) \lambda_{s}^{H} d s\right]\right)^{1 / 2} \\
& \quad<+\infty\} .
\end{aligned}
$$

Now, we suppose that $f\left(t,\left(\lambda^{B}, \lambda^{H}\right), y, z, y^{\prime}, z^{\prime}, \xi, \eta, \xi^{\prime}, \eta^{\prime}\right):[0, T] \times[0,+\infty)^{2} \times \Omega \times \mathbb{R}^{4} \times$ $S_{\mathbb{G}}^{2}(0, T+K ; \mathbb{R}) \times L_{\mathbb{G}}^{2}(0, T+K ; \mathbb{R}) \times S_{\mathbb{G}}^{2}(0, T+K ; \mathbb{R}) \times L_{\mathbb{G}}^{2}(0, T+K ; \mathbb{R}) \rightarrow L^{2}\left(\mathcal{G}_{t} ; \mathbb{R}\right)$ are two progressive functions satisfying the non-Lipschitz condition of the form

(H1) $f\left(t,\left(\lambda^{B}, \lambda^{H}\right), 0,0,0,0,0,0,0,0\right) \in L_{\mathbb{G}}^{2}(0, T+K ; \mathbb{R})$.

(H2) For every $y, y^{\prime}, \bar{y}, \bar{y}^{\prime}, z, z^{\prime}, \bar{z}, \bar{z}^{\prime} \in \mathbb{R}, \xi, \xi^{\prime}, \bar{\xi}_{\xi}, \bar{\xi}^{\prime} \in S_{\mathbb{G}}^{2}(t, T+K ; \mathbb{R})$,

$\eta, \eta^{\prime}, \bar{\eta}, \bar{\eta}^{\prime} \in L_{\mathbb{G}}^{2}(t, T+K ; \mathbb{R}) d t \times d \mathbb{P}$ a.e., $r, r^{\prime} \in[t, T+K]$, we have

$$
\begin{aligned}
& \left|f\left(t,\left(\lambda^{B}, \lambda^{H}\right), y, z, y^{\prime}, z^{\prime}, \xi_{r}, \eta_{r^{\prime}}, \xi_{r^{\prime}}^{\prime}, \eta_{r^{\prime}}^{\prime}\right)-f\left(s,\left(\lambda^{B}, \lambda^{H}\right), \bar{y}, \bar{z}, \bar{y}^{\prime}, \bar{z}^{\prime}, \bar{\xi}_{r}, \bar{\eta}_{r^{\prime}}, \bar{\xi}_{r}^{\prime}, \bar{\eta}_{r^{\prime}}^{\prime}\right)\right| \\
& \leq C \\
& \quad+\left(|y-\bar{y}|+|z(0)-\bar{z}(0)| \sqrt{\lambda^{B}}+\sqrt{\left.\int_{\mathbb{R}_{0}}|z(x)-\bar{z}(x)|^{2} q(d x) \sqrt{\lambda^{H}}\right)}\right. \\
& \quad+\left(\left|y^{\prime}-\bar{y}^{\prime}\right|+\left|z^{\prime}(0)-\bar{z}^{\prime}(0)\right| \sqrt{\lambda^{B}}+\sqrt{\int_{\mathbb{R}_{0}}\left|z^{\prime}(x)-\bar{z}^{\prime}(x)\right|^{2} q(d x)} \sqrt{\lambda^{H}}\right) \\
& \quad+E^{\mathcal{G} t}\left(\left|\xi_{r}-\bar{\xi}_{r}\right|+\left|\eta_{r^{\prime}}(0)-\bar{\eta}_{r^{\prime}}(0)\right| \sqrt{\lambda_{r^{\prime}}^{B}}+\sqrt{\int_{\mathbb{R}_{0}}\left|\eta_{r^{\prime}}(x)-\bar{\eta}_{r^{\prime}}(x)\right|^{2} q(d x)} \sqrt{\lambda_{r^{\prime}}^{H}}\right) \\
& \left.\left.\quad+\sqrt{\int_{\mathbb{R}_{0}}\left|\eta_{r^{\prime}}^{\prime}(x)-\bar{\xi}_{r}^{\prime}\right|+\left|\eta_{r^{\prime}}^{\prime}(0)-\bar{\eta}_{r^{\prime}}^{\prime}(x)\right|^{2} q(d x)} \sqrt{\lambda_{r^{\prime}}^{B}} \sqrt{\lambda_{r^{\prime}}^{H}}\right)\right],
\end{aligned}
$$

where $C>0$ is a constant. 
Liu and Dai Advances in Difference Equations

(2020) 2020:621

Page 6 of 12

3 An existence and uniqueness result for MF-ABSDE

The aim of this section is to seek out a pair of processes $(Y(t), Z(t)) \in S_{\mathbb{G}}^{2}(0, T+K ; \mathbb{R}) \times$ $L_{\mathbb{G}}^{2}(0, T+K ; \mathbb{R})$ satisfying the mean-field anticipated BSDE (3). According to Lu and Ben [11], Lemma 4, we can easily draw the following conclusion.

Lemma 4 Given a terminal condition $\xi \in L^{2}\left(\Omega, \mathcal{F}_{T}, P\right)$ and $f(t, \cdot)$ satisfy $E \int_{0}^{T}\left|f_{0}(t)\right|^{2} d t<$ $+\infty, Y_{t} \in S_{\mathbb{G}}^{2}(0, T+K ; \mathbb{R})$ and $Z_{t} \in L_{\mathbb{G}}^{2}(0, T+K ; \mathbb{R})$ is a pair of processes satisfying the following mean-field BSDE:

$$
\begin{aligned}
Y_{t} & =\xi+\int_{t}^{T} E^{\prime} f_{0}(s) d s-\int_{t}^{T} \int_{\mathbb{R}} Z_{s}(x) \mu(d s, d x) \\
& =\xi+\int_{t}^{T} E^{\prime} f_{0}(s) d s-\int_{t}^{T} Z_{s}(0) d B_{s}-\int_{t}^{T} \int_{\mathbb{R}_{0}} Z_{s}(x) \tilde{H}(d s, d x),
\end{aligned}
$$

for $\beta>0$, we have

$$
\begin{aligned}
& \left|Y_{0}\right|^{2}+E\left[\int_{0}^{T} \frac{\beta}{2} e^{\beta s}\left|Y_{s}\right|^{2} d s+\int_{0}^{T} \int_{\mathbb{R}} e^{\beta s}\left|Z_{s}\right|^{2}(x) \Lambda(d s, d x)\right] \\
& \leq E\left[|\xi|^{2} e^{\beta T}+\frac{2}{\beta} \int_{0}^{T} e^{\beta s}\left|E^{\prime} f_{0}(s)\right|^{2} d s\right] .
\end{aligned}
$$

Lemma 5 Let $f(t, \cdot)$ satisfy $(\mathrm{H} 1)$ and $(\mathrm{H} 2)$ for each $t \in[0, T]$. And suppose that $\left(y_{t}^{(k)}, z_{t}^{(k)}\right)$, $\left(y_{t}^{\prime(k)}, z_{t}^{(k)}\right) \in \mathbb{R}^{2},\left(y_{t+u(t)}^{(k)}, z_{t+v(t)}^{(k)}\right),\left(y_{t+u(t)}^{\prime(k)}, z_{t+v(t)}^{(k)}\right) \in S_{\mathbb{G}}^{2}(0, T+K ; \mathbb{R}) \times L_{\mathbb{G}}^{2}(0, T+K ; \mathbb{R})$ and $f(t, \cdot):$ $[0, T] \times[0,+\infty)^{2} \times \Omega \times \mathbb{R}^{4} \times S_{\mathbb{G}}^{2}(0, T+K ; \mathbb{R}) \times L_{\mathbb{G}}^{2}(0, T+K ; \mathbb{R}) \times S_{\mathbb{G}}^{2}(0, T+K ; \mathbb{R}) \times L_{\mathbb{G}}^{2}(0, T+$ $K ; \mathbb{R}) \rightarrow \mathbb{R}, k=1,2$, we get

$$
\begin{aligned}
& E^{\mathcal{G}_{t}}\left[\int _ { t } ^ { T } e ^ { \beta s } \left(E^{\prime \mathcal{G}_{t}} f\left(s, \lambda_{s}, y_{s}^{(1)}, z_{s}^{(1)}, y_{s}^{(1)}, z_{s}^{\prime(1)}, y_{s+u(s)}^{(1)}, z_{s+\nu(s)}^{(1)}, y_{s+u(s)}^{(1)}, z_{s+v(s)}^{(1)}\right)\right.\right. \\
&\left.\left.\quad-E^{\prime \mathcal{G}_{t}} f\left(s, \lambda_{s}, y_{s}^{(2)}, z_{s}^{(2)}, y_{s}^{(2)}, z_{s}^{(2)}, y_{s+u(s)}^{(2)}, z_{s+\nu(s)}^{(2)}, y_{s+u(s)}^{(2)}, z_{s+\nu(s)}^{(2)}\right)\right) d s\right]^{2} \\
& \leq 24 C^{2}(1+L)(T-t) E^{\mathcal{G}_{t}}\left[\int_{t}^{T+K} e^{\beta s}\left|y_{s}^{(1)}-y_{s}^{(2)}\right|^{2} d s\right. \\
&\left.+\int_{t}^{T+K} \int_{\mathbb{R}} e^{\beta s}\left|z_{t}^{(1)}(x)-z_{t}^{(2)}(x)\right|^{2} \Lambda(d s, d x)\right] .
\end{aligned}
$$

Proof Using conditions (A1)-(A2) and (H2), we get

$$
\begin{aligned}
E^{\mathcal{G}_{t}}\left[\int _ { t } ^ { T } e ^ { \beta s } \left(E^{\prime \mathcal{G}_{t}} f\left(s, \lambda_{s}, y_{s}^{(1)}, z_{s}^{(1)}, y_{s}^{\prime(1)}, z_{s}^{(1)}, y_{s+u(s)}^{(1)}, z_{s+\nu(s)}^{(1)}, y_{s+u(s)}^{(1)}, z_{s+\nu(s)}^{\prime(1)}\right)\right.\right. \\
\left.\left.-\quad E^{\prime \mathcal{G}_{t}} f\left(s, \lambda_{s}, y_{s}^{(2)}, z_{s}^{(2)}, y_{s}^{\prime(2)}, z_{s}^{(2)}, y_{s+u(s)}^{(2)}, z_{s+\nu(s)}^{(2)}, y_{s+u(s)}^{(2)}, z_{s+\nu(s)}^{(2)}\right)\right) d s\right]^{2} \\
\leq C^{2} E^{\mathcal{G}_{t}}\left\{\int _ { t } ^ { T } e ^ { \beta s } \left[\left(\left|y_{s}^{(1)}-y_{s}^{(2)}\right|+\left|z_{s}^{(1)}(0)-z_{s}^{(2)}(0)\right| \sqrt{\lambda_{s}^{B}}\right.\right.\right. \\
\left.\quad+\sqrt{\int_{\mathbb{R}_{0}}\left|z_{s}^{(1)}(x)-z_{s}^{(2)}(x)\right|^{2} q(d x)} \sqrt{\lambda_{s}^{H}}\right)
\end{aligned}
$$




$$
\begin{aligned}
& +\left(\left|y_{s}^{\prime(1)}-y_{s}^{\prime(2)}\right|+\left|z_{s}^{(1)}(0)-z_{s}^{(2)}(0)\right| \sqrt{\lambda_{s}^{B}}+\sqrt{\int_{\mathbb{R}_{0}}\left|z_{s}^{\prime(1)}(x)-z_{s}^{\prime(2)}(x)\right|^{2} q(d x)} \sqrt{\lambda_{s}^{H}}\right) \\
& +E^{\mathcal{G}_{s}}\left(\left|y_{s+u(s)}^{(1)}-y_{s+u(s)}^{(2)}\right|+\left|z_{s+v(s)}^{(1)}(0)-z_{s+v(s)}^{(2)}(0)\right| \sqrt{\lambda_{s+v(s)}^{B}}\right. \\
& \left.+\sqrt{\int_{\mathbb{R}_{0}}\left|z_{s+v(s)}^{(1)}(x)-z_{s+v(s)}^{(2)}(x)\right|^{2} q(d x)} \sqrt{\lambda_{s+v(s)}^{H}}\right) \\
& +E^{\prime \mathcal{G}_{s}}\left(\left|y_{s+u(s)}^{\prime(1)}-y_{s+u(s)}^{\prime(2)}\right|+\left|z_{s+v(s)}^{\prime(1)}(0)-z_{s+v(s)}^{\prime(2)}(0)\right| \sqrt{\lambda_{s+v(s)}^{B}}\right. \\
& \left.\left.\left.+\sqrt{\int_{\mathbb{R}_{0}}\left|z_{s+v(s)}^{(1)}(x)-z_{s+v(s)}^{\prime(2)}(x)\right|^{2} q(d x)} \sqrt{\lambda_{s+v(s)}^{H}}\right)\right]^{2}\right\} d s \\
& \leq 24 C^{2}(T-t) E^{\mathcal{G}_{t}}\left\{\int _ { t } ^ { T } e ^ { \beta s } \left[\left(\left|y_{s}^{(1)}-y_{s}^{(2)}\right|^{2}\right.\right.\right. \\
& \left.+\left|z_{s}^{(1)}(0)-z_{s}^{(2)}(0)\right|^{2} \lambda_{s}^{B}+\int_{\mathbb{R}_{0}}\left|z_{s}^{(1)}(x)-z_{s}^{(2)}(x)\right|^{2} q(d x) \lambda_{s}^{H}\right) \\
& +E^{\mathcal{G}_{s}}\left(\left|y_{s+u(s)}^{(1)}-y_{s+u(s)}^{(2)}\right|^{2}+\left|z_{s+v(s)}^{(1)}(0)-z_{s+v(s)}^{(2)}(0)\right|^{2} \lambda_{s+\nu(s)}^{B}\right. \\
& \left.\left.+\int_{\mathbb{R}_{0}}\left|z_{s}^{(1)}(x)-z_{s}^{(2)}(x)\right|^{2} q(d x) \lambda_{s+v(s)}^{H}\right)\right] d s \\
& \leq 24 C^{2}(1+L)(T-t) E^{\mathcal{G} t}\left[\int_{t}^{T+K} e^{\beta s}\left|y_{s}^{(1)}-y_{s}^{(2)}\right|^{2} d s\right. \\
& \left.+\int_{t}^{T+K} \int_{\mathbb{R}} e^{\beta s}\left|z_{s}^{(1)}(x)-z_{s}^{(2)}(x)\right|^{2} \Lambda(d s, d x)\right] .
\end{aligned}
$$

Theorem 6 Suppose that $\xi(t) \in S_{\mathbb{G}}^{2}(T, T+K ; \mathbb{R})$ and $\eta(t) \in L_{\mathbb{G}}^{2}(T, T+K ; \mathbb{R}),(\mathrm{H} 1)$ and $(\mathrm{H} 2)$ hold, $u(\cdot)$ and $v(\cdot)$ satisfy (A1) and (A2). The MF-ABSDE (3) has a unique solution $(Y, Z)$, i.e., we have $Y \in S_{\mathbb{G}}^{2}(0, T+K ; \mathbb{R}), Z \in L_{\mathbb{G}}^{2}(0, T+K ; \mathbb{R})$ satisfying $M F-A B S D E$ (3).

Proof We structure a norm on the space $S_{\mathbb{G}}^{2}(0, T+K ; \mathbb{R}) \times L_{\mathbb{G}}^{2}(0, T+K ; \mathbb{R})$ which is equivalent to the following norm:

$$
\|(g, h)\|_{\beta}=\left(E \int_{0}^{T+K} e^{\beta s}\left|g_{s}\right|^{2} d s+E \int_{0}^{T+K} \int_{\mathbb{R}} e^{\beta s}\left|h_{s}(x)\right|^{2} \Lambda(d s, d x)\right)^{1 / 2} .
$$

We consider the following MF-ABSDE driven by Lévy noises:

$$
\left\{\begin{aligned}
Y_{t}= & \xi_{T}+\int_{t}^{T} E^{\prime}\left[f\left(t, \lambda_{t}, Y_{t}, Z_{t}, Y_{t}^{\prime}, Z_{t}^{\prime}, Y_{t+u(t)}, Z_{t+v(t)}, Y_{t+u(t)}^{\prime}, Z_{t+v(t)}^{\prime}\right)\right] d t \\
& -\int_{t}^{T} Z_{t}(x) \mu(d t, d x), \quad t \in[0, T] \\
Y_{t}= & \xi_{t}, \quad t \in[T, T+K] \\
Z_{t}= & \eta_{t}, \quad t \in[T, T+K] .
\end{aligned}\right.
$$

For two any elements $\left(y^{(1)}, z^{(1)}\right),\left(y^{(2)}, z^{(2)}\right) \in S_{\mathbb{G}}^{2}(0, T+K ; \mathbb{R}) \times L_{\mathbb{G}}^{2}(0, T+K ; \mathbb{R})$, we define a mapping $\Psi: S_{\mathbb{G}}^{2}(0, T+K ; \mathbb{R}) \times L_{\mathbb{G}}^{2}(0, T+K ; \mathbb{R}) \rightarrow S_{\mathbb{G}}^{2}(0, T+K ; \mathbb{R}) \times L_{\mathbb{G}}^{2}(0, T+K ; \mathbb{R})$, that is, $\Psi[(y, z)]=(Y, Z)$. We introduce the mapping $\left(Y^{(1)}, Z^{(1)}\right)=\Psi\left[\left(y^{(1)}, z^{(1)}\right)\right]$ and $\left(Y^{(2)}, Z^{(2)}\right)=$ 
$\Psi\left[\left(y^{(2)}, z^{(2)}\right)\right]: S_{\mathbb{G}}^{2}(0, T+K ; \mathbb{R}) \times L_{\mathbb{G}}^{2}(0, T+K ; \mathbb{R}) \rightarrow S_{\mathbb{G}}^{2}(0, T+K ; \mathbb{R}) \times L_{\mathbb{G}}^{2}(0, T+K ; \mathbb{R})$ through MF-ABSDEs (7). We put $\hat{Y}=Y^{(1)}-Y^{(2)}, \hat{Z}=Z^{(1)}-Z^{(2)}, \hat{y}=y^{(1)}-y^{(2)}, \hat{z}=z^{(1)}-z^{(2)}$, and

$$
\begin{aligned}
{E^{\prime}}^{\prime \mathcal{G}_{t}} \hat{f}(s)= & E^{\prime \mathcal{G}_{t}} f\left(s, \lambda_{s}, y_{s}^{(1)}, z_{s}^{(1)}, y_{s}^{(1)}, z_{s}^{(1)}, y_{s+u(s)}^{(1)}, z_{s+\nu(s)}^{(1)}, y_{s+u(s)}^{(1)}, z_{s+\nu(s)}^{(1)}\right) \\
& -E^{\prime \mathcal{G}_{t}} f\left(s, \lambda_{s}, y_{s}^{(2)}, z_{s}^{(2)}, y_{s}^{(2)}, z_{s}^{(2)}, y_{s+u(s)}^{(2)}, z_{s+\nu(s)}^{(2)}, y_{s+u(s)}^{(2)}, z_{s+\nu(s)}^{(2)}\right) .
\end{aligned}
$$

Then we have

$$
\left\{\begin{array}{l}
d \hat{Y}_{t}=E^{\prime} \mathcal{G}_{t} \hat{f}(t) d t-\hat{Z}_{t}(x) \mu(d t, d x), \quad t \in[0, T] \\
\hat{Y}_{t}=0, \quad t \in[T, T+K] \\
\hat{Z}_{t}=0, \quad t \in[T, T+K]
\end{array}\right.
$$

Now we will prove that $Y \in S_{\mathbb{G}}^{2}(0, T+K ; \mathbb{R}), Z \in L_{\mathbb{G}}^{2}(0, T+K ; \mathbb{R})$ solves MF-ABSDE (3) if and only if it is a fixed point of $\Psi$.

By Lemma 4, we get

$$
E\left[\int_{0}^{T} \frac{\beta}{2} e^{\beta t}\left|\hat{Y}_{s}\right|^{2} d s+\int_{0}^{T} \int_{\mathbb{R}} e^{\beta t}\left|\hat{Z}_{s}\right|^{2}(x) \Lambda(d s, d x)\right] \leq \frac{2}{\beta} E\left(\int_{0}^{T} e^{\beta s}\left|E^{\prime} \mathcal{G}_{s} \hat{f}(s)\right|^{2} d s\right) .
$$

Using the fact that $u(x), v(x)$ satisfy (A1) and (A2), by Lemma 5 , we get

$$
\begin{aligned}
E[ & \left.\int_{0}^{T} \frac{\beta}{2} e^{\beta s}\left|\hat{Y}_{s}\right|^{2} d s+\int_{0}^{T} \int_{\mathbb{R}} e^{\beta s}\left|\hat{Z}_{s}\right|^{2}(x) \Lambda(d s, d x)\right] \\
\leq & \frac{2}{\beta} E\left\{\int _ { 0 } ^ { T } e ^ { \beta s } \left[E^{\prime \mathcal{G}} f\left(s, \lambda_{s}, y_{s}^{(1)}, z_{s}^{(1)}, y_{s}^{(1)}, z_{s}^{(1)}, y_{s+u(s)}^{(1)}, z_{s+\nu(s)}^{(1)}, y_{s+u(s)}^{(1)}, z_{s+v(s)}^{(1)}\right)\right.\right. \\
& \left.\left.-E^{\prime \mathcal{G}} f\left(s, \lambda_{s}, y_{s}^{(2)}, z_{s}^{(2)}, y_{s}^{(2)}, z_{s}^{(2)}, y_{s+u(s)}^{(2)}, z_{s+\nu(s)}^{(2)}, y_{s+u(s)}^{(2)}, z_{s+\nu(s)}^{(2)}\right)\right]\right\} \\
\leq & \frac{48 C^{2}(1+L)(T-t)}{\beta} E\left[\int_{0}^{T+K} e^{\beta s}\left|\bar{y}_{s}\right|^{2} d s+\int_{0}^{T+K} \int_{\mathbb{R}} e^{\beta s}\left|\bar{z}_{s}(x)\right|^{2} \Lambda(d s, d x)\right],
\end{aligned}
$$

that is,

$$
\begin{aligned}
& E\left[\int_{0}^{T+K} e^{\beta s}\left|\bar{Y}_{s}\right|^{2} d s+\int_{0}^{T+K} \int_{\mathbb{R}} e^{\beta s}\left|\bar{Z}_{s}\right|^{2}(x) \Lambda(d s, d x)\right] \\
& \quad \leq \frac{48 C^{2} T(1+L)}{\beta} E\left[\int_{0}^{T+K} e^{\beta s}\left|\bar{y}_{s}\right|^{2} d s+\int_{0}^{T+K} \int_{\mathbb{R}} e^{\beta s}\left|\bar{z}_{s}(x)\right|^{2} \Lambda(d s, d x)\right] .
\end{aligned}
$$

Let $\beta=96 C^{2} T(1+L)+2$, it is very easy for us to find $\|(\hat{Y}, \hat{Z})\|_{\beta} \leq \frac{1}{\sqrt{2}}\|(\hat{y}, \hat{z})\|_{\beta}$. Hence we see that this mapping $\Psi$ is a strict contraction on $S_{\mathbb{G}}^{2}(0, T+K ; \mathbb{R}) \times L_{\mathbb{G}}^{2}(0, T+K ; \mathbb{R})$. It follows by the fixed point theorem that $\Psi$ has a unique fixed point, that is, the MF-ABSDE (3) has a unique solution $\left(Y_{t}, Z_{t}\right) \in S_{\mathbb{G}}^{2}(0, T+K ; \mathbb{R}) \times L_{\mathbb{G}}^{2}(0, T+K ; \mathbb{R})$ on $[0, T+K]$ such that $\Psi(Y, Z)=(Y, Z)$. The proof is complete.

Remark 7 In fact, we almost surely get the solution of the mean-field anticipated BSDE (3) by the Picard iterative sequence. 
We suppose that $\left(Y_{t}^{n}, Z_{t}^{n}\right)$ is the sequence defined recursively by $\left(Y_{t}^{0}=0, Z_{t}^{0}=0\right)$ and

$$
\left\{\begin{aligned}
d Y_{t}^{n+1}= & -E^{\prime}\left[f\left(t, \lambda_{t}, Y_{t}^{n}, Z_{t}^{n}, Y_{t}^{\prime n}, Z_{t}^{\prime n}, Y_{t+u(t)}^{n}, Z_{t+v(t)}^{n}, Y_{t+u(t)}^{\prime n}, Z_{t+v(t)}^{\prime n}\right)\right] d t \\
& +Z_{t}^{n+1}(x) \mu(d t, d x), \quad t \in[0, T], \\
Y_{t}^{n+1}= & \xi_{t}, \quad t \in[T, T+K], \\
Z_{t}^{n+1}= & \eta_{t}, \quad t \in[T, T+K] .
\end{aligned}\right.
$$

Then the sequence $\left(Y_{t}^{n}, Z_{t}^{n}\right)$ converges to $(Y(\cdot), Y(\cdot))$ when $n \rightarrow+\infty$.

In fact, suppose $\left(Y_{t}^{n}, Z_{t}^{n}\right)$ is the sequence defined recursively by (10). By (9)

$$
\left\|Y_{t}^{n+1}-Y_{t}^{n}\right\|^{2}+\left\|Z_{t}^{n+1}-Z_{t}^{n}\right\|^{2} \leq\left(\frac{48 C^{2} T(1+L)}{\beta}\right)^{n}\left(\left\|Y_{t}^{1}-Y_{t}^{0}\right\|^{2}+\left\|Z_{t}^{1}-Z_{t}^{0}\right\|^{2}\right),
$$

choosing $\beta>48 C^{2} T(1+L)$, we find that $\left\{Y_{t}^{n}\right\}$ is a Cauchy sequence in $S_{\mathbb{G}}^{2}(0, T+K ; \mathbb{R})$ and $\left\{Z_{t}^{n}\right\}$ is a Cauchy sequence in $L_{\mathbb{G}}^{2}(0, T+K ; \mathbb{R})$ and the result follows.

\section{Comparison theorem}

In this section, we will study a kind of mean-field anticipated BSDE driven by timechanging Lévy noises. Let $\left(Y^{(1)}, Z^{(1)}\right),\left(Y^{(2)}, Z^{(2)}\right)$ be, respectively, solutions for the following two one-dimensional mean-field anticipated BSDE:

$$
\left\{\begin{aligned}
Y_{t}^{(k)}= & \xi_{T}+\int_{t}^{T} E^{\prime} f^{(k)}\left(s, \lambda_{s}, Y_{s}^{(k)}, Z_{s}^{(k)}, Y_{s+u(s)}^{\prime(k)}, Z_{s+v(s)}^{\prime(k)}\right) d s \\
& -\int_{t}^{T} \int_{\mathbb{R}} Z_{s}^{(k)}(x) \mu(d s, d x), \quad t \in[0, T] \\
Y_{t}^{(k)}= & \xi_{t}^{(k)}, \quad t \in[T, T+K] ; \\
Z_{t}^{(k)}= & \eta_{t}^{(k)}, \quad t \in[T, T+K] .
\end{aligned}\right.
$$

That is,

$$
\left\{\begin{aligned}
-d Y_{t}^{(k)}= & E^{\prime} f^{(k)}\left(t, \lambda_{t}, Y_{t}^{(k)}, Z_{t}^{(k)}, Y_{t+u(t)}^{\prime(k)}, Z_{t+v(t)}^{(k)}\right) d t-Z_{t}^{(k)}(0) d B_{t} \\
& \quad-\int_{\mathbb{R}_{0}} Z_{t}^{(k)}(x) \tilde{H}(d t, d x), \quad t \in[0, T] ; \\
Y_{t}^{(k)}=\xi_{t}^{(k)}, \quad t \in[T, T+K] ; & t(k)=\eta_{t}^{(k)}, \quad t \in[T, T+K],
\end{aligned}\right.
$$

where $k=1,2$.

Theorem 8 Assume that $f^{(1)}(t, \cdot), f^{(2)}(t, \cdot)$ satisfy $(\mathrm{H} 1)-(\mathrm{H} 2), \xi_{t}^{(1)}, \xi_{t}^{(2)} \in S_{\mathbb{G}}^{2}(T, T+K ; \mathbb{R})$ and (A1), (A2) hold for $u(\cdot), v(\cdot)$. Assume:

(H3) One of the two coefficients is independent of $z^{\prime}$.

(H4) One of the two coefficients is nondecreasing in $y^{\prime}$.

Moreover, we suppose that

(H5) $\xi_{t}^{(1)} \leq \xi_{t}^{(2)}, t \in[T, T+K] \mathbb{P}$-a.s.

(H6) $f^{(1)}(t, \cdot) \leq f^{(2)}(t, \cdot) \mathbb{P}$-a.s.

It is then true that $Y_{t}^{(1)} \leq Y_{t}^{(2)} \mathbb{P}$-a.s.

Proof Without loss of generality, we assume that $f^{(1)}(t, \cdot)$ is nondecreasing in $y^{\prime}$ and $f^{(2)}(t, \cdot)$ is independent of $z^{\prime}$. For notational simplicity we use $\hat{Y}_{t}=Y_{t}^{(1)}-Y_{t}^{(2)}, \hat{Z}_{t}=Z_{t}^{(1)}-Z_{t}^{(2)}, \hat{\xi}_{t}=$ 
$\xi_{t}^{(1)}-\xi_{t}^{(2)}$; then the pair $(Y, Z)$ can be regarded as the solution to the mean-field anticipated BSDE

$$
\begin{aligned}
-d \hat{Y}_{t}= & E^{\prime}\left[f^{(1)}\left(t, \lambda_{t}, Y_{t}^{(1)}, Z_{t}^{(1)}, Y_{t+u(t)}^{\prime(1)}, Z_{t+v(t)}^{(1)}\right)-f^{(2)}\left(t, \lambda_{t}, Y_{t}^{(2)}, Z_{t}^{(2)}, Y_{t+u(t)}^{\prime(2)}\right)\right] d t \\
& -\hat{Z}_{t}(x) \mu(d t, d x), \quad t \in[0, T] .
\end{aligned}
$$

From the Itô formula applied from $s=t$ to $s=T$ on $\left(\hat{Y}_{t}^{+}\right)^{2}$, it follows that

$$
\begin{aligned}
& E^{\mathcal{G}_{t}}\left|\hat{Y}_{t}^{+}\right|^{2}+E^{\mathcal{G}_{t}} \int_{t}^{T} \int_{\mathbb{R}} 1_{\left\{\hat{Y}_{s}>0\right\}}\left|\hat{Z}_{s}(x)\right|^{2} \Lambda(d s, d x) \\
& =2 E^{\mathcal{G}_{t}} \int_{t}^{T} \hat{Y}_{s}^{+} E^{\prime}\left[f^{(1)}\left(s, \lambda_{s}, Y_{s}^{(1)}, Z_{s}^{(1)}, Y_{s+u(s)}^{\prime(1)}, Z_{s+v(s)}^{(1)}\right)-f^{(2)}\left(s, \lambda_{s}, Y_{s}^{(2)}, Z_{s}^{(2)}, Y_{s+u(s)}^{\prime(2)}\right)\right] d s \\
& =2 E^{\mathcal{G}_{t}} \int_{t}^{T} \hat{Y}_{s}^{+} E^{\prime}\left[f^{(1)}\left(s, \lambda_{s}, Y_{s}^{(1)}, Z_{s}^{(1)}, Y_{s+u(s)}^{\prime(1)}, Z_{s+v(s)}^{(1)}\right)\right. \\
& \left.-f^{(1)}\left(s, \lambda_{s}, Y_{s}^{(1)}, Z_{s}^{(1)}, Y_{s+u(s)}^{\prime(2)}, Z_{s+v(s)}^{(1)}\right)\right] d s \\
& +2 E^{\mathcal{G}_{t}} \int_{t}^{T} \hat{Y}_{s}^{+} E^{\prime}\left[f^{(1)}\left(s, \lambda_{s}, Y_{s}^{(1)}, Z_{s}^{(1)}, Y_{s+u(s)}^{\prime(2)}, Z_{t+v(s)}^{(1)}\right)-f^{(2)}\left(s, \lambda_{s}, Y_{s}^{(2)}, Z_{s}^{(2)}, Y_{s+u(s)}^{\prime(2)}\right)\right] d s \\
& \leq 2 E^{\mathcal{G} t} \int_{t}^{T} \hat{Y}_{s}^{+} E^{\prime}\left[f^{(1)}\left(s, \lambda_{s}, Y_{s}^{(1)}, Z_{s}^{(1)}, Y_{s+u(s)}^{\prime(1)}, Z_{s+v(s)}^{(1)}\right)\right. \\
& \left.-f^{(1)}\left(s, \lambda_{s}, Y_{s}^{(1)}, Z_{s}^{(1)}, Y_{s+u(s)}^{\prime(2)}, Z_{s+\nu(s)}^{(1)}\right)\right] d s \\
& +2 E^{\mathcal{G} t} \int_{t}^{T} \hat{Y}_{s}^{+} E^{\prime}\left[f^{(2)}\left(s, \lambda_{s}, Y_{s}^{(1)}, Z_{s}^{(1)}, Y_{s+u(s)}^{\prime(2)}\right)-f^{(2)}\left(s, \lambda_{s}, Y_{s}^{(2)}, Z_{s}^{(2)}, Y_{s+u(s)}^{\prime(2)}\right)\right] d s .
\end{aligned}
$$

From assumptions (H1)-(H2) and (A1)-(A2), we obtain

$$
\begin{aligned}
& 2 E^{\mathcal{G}_{t}} \int_{t}^{T} \hat{Y}_{s}^{+} E^{\prime}\left[f^{(1)}\left(s, \lambda_{s}, Y_{s}^{(1)}, Z_{s}^{(1)}, Y_{s+u(s)}^{\prime(1)}, Z_{s+\nu(s)}^{\prime(1)}\right)-f^{(1)}\left(s, \lambda_{s}, Y_{s}^{(1)}, Z_{s}^{(1)}, Y_{s+u(s)}^{\prime(2)}, Z_{s+\nu(s)}^{\prime(1)}\right)\right] d s \\
& \quad \leq 2 C E^{\mathcal{G}_{t}} \int_{t}^{T} \hat{Y}_{s}^{+} E^{\prime}\left(Y_{s+u(s)}^{\prime(1)}-Y_{s+u(s)}^{\prime(2)}\right)^{+} d s \\
& \quad \leq 2 C L \int_{t}^{T} E^{\mathcal{G}_{t}}\left|\hat{Y}_{s}^{+}\right|^{2} d s
\end{aligned}
$$

and

$$
\begin{aligned}
& 2 E^{\mathcal{G}_{t}} \int_{t}^{T} \hat{Y}_{s}^{+} E^{\prime}\left[f^{(2)}\left(s, \lambda_{s}, Y_{s}^{(1)}, Z_{s}^{(1)}, Y_{s+u(s)}^{\prime(2)}\right)-f^{(2)}\left(s, \lambda_{s}, Y_{s}^{(2)}, Z_{s}^{(2)}, Y_{s+u(s)}^{\prime(2)}\right)\right] d t \\
& \leq 2 C E^{\mathcal{G}_{t}} \int_{t}^{T} \hat{Y}_{s}^{+} E^{\prime}\left(\left|\hat{Y}_{s}\right|+|\hat{Z}(0)| \sqrt{\lambda_{s}^{B}}+\sqrt{\int_{\mathbb{R}_{0}}\left|\hat{Z}_{s}(x)\right|^{2} q(d x) \sqrt{\lambda_{s}^{H}}}\right) d s \\
& \quad \leq 2 C \int_{t}^{T} E^{\mathcal{G}_{s}}\left|\hat{Y}_{s}\right|^{2} d s+2 C E^{\mathcal{G}_{t}} \int_{t}^{T} \hat{Y}_{s}^{+} E^{\prime}\left(\left|\hat{Z}_{s)}^{\prime}(0)\right| \sqrt{\lambda_{s}^{B}}+\sqrt{\int_{\mathbb{R}_{0}}\left|\hat{Z}_{s}^{\prime}(x)\right|^{2} q(d x)} \sqrt{\lambda_{s}^{H}}\right) d s \\
& \leq\left(2 C+4 C^{2}\right) \int_{t}^{T} E^{\mathcal{G}_{s}}\left|\hat{Y}_{s}^{+}\right|^{2} d s
\end{aligned}
$$




$$
\begin{gathered}
+\frac{1}{4} E^{\mathcal{G} t} \int_{t}^{T} 1_{\left\{\hat{Y}_{s}>0\right\}}\left(|\hat{Z}(0)| \sqrt{\lambda_{s}^{B}}+\sqrt{\int_{\mathbb{R}_{0}}\left|\hat{Z}_{s}(x)\right| q(d x)} \sqrt{\lambda_{s}^{H}}\right)^{2} d s \\
\leq\left(2 C+4 C^{2}\right) \int_{t}^{T} E^{\mathcal{G}_{s}}\left|\hat{Y}_{s}^{+}\right|^{2} d s+\frac{1}{2} E^{\mathcal{G}_{t}} \int_{t}^{T} \int_{\mathbb{R}} 1_{\left\{\hat{Y}_{s}>0\right\}}\left|\hat{Z}_{s}(x)\right|^{2} \Lambda(d s, d x),
\end{gathered}
$$

where we use the fact that $2 a b \leq c a^{2}+\frac{1}{c} b^{2}, c>0$. We get

$$
\begin{aligned}
E^{\mathcal{G}_{t}}\left|\hat{Y}_{t}^{+}\right|^{2} \leq & -\frac{1}{2} E^{\mathcal{G}_{t}} \int_{t}^{T} \int_{\mathbb{R}} 1_{\left\{\hat{Y}_{s}>0\right\}}\left|\hat{Z}_{s}(x)\right|^{2} \Lambda(d s, d x) \\
& +\left(2 C+2 C L+4 C^{2}\right) \int_{t}^{T} E^{\mathcal{G}_{s}}\left|\hat{Y}_{s}^{+}\right|^{2} d s, \quad t \in[0, T] .
\end{aligned}
$$

Then from Gronwall's inequality it follows that $\hat{Y}_{t}=Y_{t}^{(1)}-Y_{t}^{(2)} \leq 0, t \in[0, T], \mathbb{P}$-a.s.

\section{Acknowledgements}

The authors would like to thank the reviewers for their valuable comments and suggestions, which helped to improve its presentation overall.

\section{Funding}

The research of Youxin Liu is supported by Foundation for Outstanding Young in Higher Education of Anhui of China (gxyq2020119), WHIT Mathematical Modeling Master studio and Anhui University Natural Science Research Project (KJ2019A0976).

\section{Availability of data and materials}

Not applicable.

\section{Competing interests}

The authors declare that they have no competing interests.

\section{Authors' contributions}

The two authors provided the basic idea of this work. The corresponding author YL wrote the draft of the manuscript, and the other author YD revised the manuscript. All authors read and approved the final manuscript.

\section{Author details}

${ }^{1}$ Department of Elementary Teaching, Wuhu Institute of Technology, Wuhu, China. ${ }^{2}$ School of Science, Nanjing University of Science and Technology, Nanjing, China. ${ }^{3}$ School of Mathematics and Statistics, Anhui Normal University, Wuhu, China.

\section{Publisher's Note}

Springer Nature remains neutral with regard to jurisdictional claims in published maps and institutional affiliations.

Received: 27 March 2020 Accepted: 7 October 2020 Published online: 05 November 2020

\section{References}

1. Buckdahn, R., Li, J., Ma, J.: A stochastic maximum principle for general mean-field systems. Appl. Math. Optim. 74, 507-534 (2016). https://doi.org/10.1007/s00245-016-9394-9

2. Buckdahn, R., Li, J., Peng, S.: Mean-field backward stochastic differential equations and related partial differential equations. Stoch. Process. Appl. 119, 3133-3154 (2009)

3. Di Nunno, G., Sjursen, S.: BSDEs driven by time-changed Lévy noises and optimal control. Stoch. Process. Appl. 124 1679-1709 (2014)

4. Douissi, S., Wen, J., Shi, Y.: Mean-field anticipated BSDEs driven by fractional Browian motion and related stochastic control problem. Appl. Math. Comput. 355, 282-298 (2019)

5. El Karou, N., Peng, S., Quenez, M.C.: Backward stochastic differential equations finance. Math. Finance 7(1), 1-71 (1997)

6. Frei, C.: Splitting multidimensional BSDEs and finding local equilibria. Stoch. Process. Appl. 124, 2654-2671 (2014)

7. Giulia, D., Steffen, S.: BSDEs driven by time-changed Lévy noises and optimal control. Stoch. Process. Appl. 124 1679-1709 (2014)

8. Kallsen, J., Muhle-Karbe, J.: Utility maximization in affine stochastic volatility models. Int. J. Theor. Appl. Finance 13(03), 459-477 (2010)

9. Klimsiak, T.: Reflected BSDEs with monotone generator. Electron. J. Probab. 107, 1-25 (2012)

10. Liu, Y.: Applications of anticipated BSDEs driven by time-changing Lévy noises. J. Inequal. Appl. 2016, 290 (2016)

11. Liu, Y., Ren, Y.: Anticipated BSDEs driven by time-changed Lévy noises. J. Korean Stat. Soc. 44(3), 403-409 (2015)

12. Lu, W., Ren, Y.: Anticipated backward stochastic differential equations on Markov chains. Stat. Probab. Lett. 83 1711-1719 (2013) 
13. Pardoux, E., Peng, S.: Adapted solution of a backward stochastic differential equation. Syst. Control Lett. 14, 55-61 (1990)

14. Peng, S.: Nonlinear expectations and nonlinear Markov chains. Chin. Ann. Math. 26(02), 159-184 (2005)

15. Peng, S., Yang, Z.: Anticipated backward stochastic differential equations. Ann. Probab. 37, 877-902 (2009)

16. Ren, Y., Mohamed, E.O.: Generalized reflected BSDEs driven by a Lévy process and an obstacle problem for PDIEs with a nonlinear Neumann boundary condition. J. Comput. Appl. Math. 233, 2027-2043 (2010)

17. Richter, A.: Explicit solutions to quadratic BSDEs and applications to utility maximization in multivariate affine stochastic volatility models. Stoch. Process. Appl. 124, 3578-3611 (2014)

18. Wang, M., Shi, Q., Meng, Q.: Optimal control of forward-backward stochastic jump-diffusion differential systems with observation noises: stochastic maximum principle. Asian J. Control 1-14 (2019). https://doi.org/10.1002/asjc.2272

19. Wang, W: Optimal control of backward doubly stochastic system. IET Control Theory Appl. 13, 1844-1854 (2019)

20. Xu, X: Necessary and sufficient condition for the comparison theorem of multidimensional anticipated backward stochastic differential equations. Sci. China Math. 54, 301-310 (2011)

21. $\mathrm{Yu}, \mathrm{Z}$ : The stochastic maximum principle for optimal control problems of delay systems involving continuous and impulse controls. Automatica 48, 2420-2432 (2012)

22. Zhang, H., Yan, Z.: Backward stochastic optimal control with mixed deterministic controller and random controller and its applications in linear-quadratic control. Appl. Math. Comput. 369, 1-11 (2020)

\section{Submit your manuscript to a SpringerOpen ${ }^{\circ}$ journal and benefit from:}

- Convenient online submission

- Rigorous peer review

- Open access: articles freely available online

- High visibility within the field

- Retaining the copyright to your article

Submit your next manuscript at $\gg$ springeropen.com 